\title{
aniki
}

Revista Portuguesa da Imagem em Movimento

Portuguese Journal of the Moving Image

\section{Engagement Design: Designing for interaction motivations}

\section{Rogério Júnior Correia Tavares}

UFRN Arts Department - Universidade Federal do Rio Grande do Norte rogertavares@gmail.com

https://orcid.org/0000-0001-9366-2617

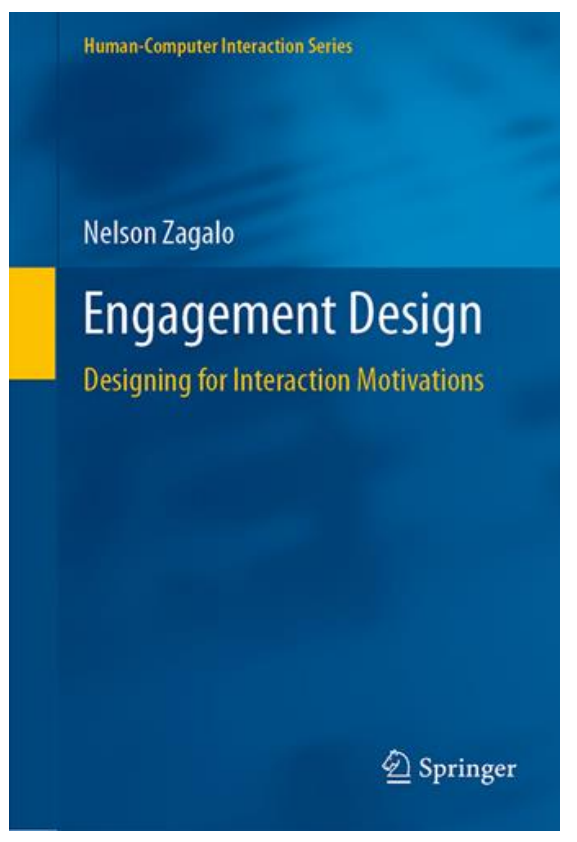

Zagalo, Nelson. 2020. Engagement Design: Designing for interaction motivations. Springer International Publishing, 161 pp. ISBN 978-3030-37085-5 (eBook)

In this book, researcher Nelson Zagalo, from the Department of Communication and Arts at University of Aveiro, Portugal, addresses the issue of engagement in digital media, focusing on digital games. The topic has not received the attention it deserves in studies on digital averages, and therefore this is an important contribution to this field.

The book belongs to Springer's Human-Computer Interaction Series, which, as the name implies, aims to address the issue of HCI. But what the name of the collection does not reveal is that more than 130 books have been published in this series in more than 15 years and this is probably the first publication to give high priority to this issue. Certainly, there are several books dealing with the user experience, one of the main objects of study in 
HCI, but not mainly from the point of view of engagement. HCI is a field of multidisciplinary studies that works on the development of computational technologies based on human aspects, and, in this sense, the participation of Zagalo and other humanities researchers is very important for the debate. The author has devoted several years of work to the subject of interaction, especially in subjects such as interactive narratives, interactive experience, expressiveness in games, agency, aesthetics, emotions and many others.

The word engagement has many meanings. In the Portuguese language there are two that are a close fit to the field: (1) when someone voluntarily participates in something like a political or philanthropic cause, it is said that the person is engaged in that cause, a sense we can also call participation; and (2) in the sense of dedication, when someone acts wilfully, engaging in some goal such as finishing a game, and we say the person is really engaged in it. In the case of digital media, engagement is one of the factors that leads to immersion, and it is provided by several other factors, such as mechanics, interfaces and narratives, that lead the user to engage with a certain medium and get involved. But how does this engagement happen, and how can we think on and evaluate a project in order to engage users? This is where the book leads us, through a proposed model based on three streams of engagement: progression, expression, and relationship, which I will address later.

To achieve its purpose, the 161 pages of the book present an introduction and five chapters, structured as follows: a discussion on interdisciplinarity from a historical review, the construction and use of the proposed model, and a conclusion. Along the way, the book features 26 illustrations in black and white and 62 in colour, a rich bibliography and an index.

In the introduction, the author begins with an epistemological debate on the problems of the division of knowledge, and the importance of interdisciplinarity. Through a conciliatory view, the author presents the problem through a literature review in the debate between the different sciences. From Plato to Nigel Cross, through Brockman, Pinker and Gates, this chapter encompasses polymaths, such as Athanasius Kircher and Leonardo da Vinci, and considers how the specialization of knowledge would be hard for these people who were able to act in such distinct areas. Describing the current technological context, Zagalo evokes Kevin Kelly (2010, 37), for whom "technology has domesticated us [...] We are co-evolving with our technology" (p. 2), which, in a way, echoes Neil Postman's Technopoly (1993), with the difference that in Postman we end 
up handing over our responsibilities to technology in a way. I emphasise here the importance of this debate contextualised in technology, since Zagalo's book is a contribution in the sense of not giving over our responsibilities, our culture, our capacities, to technology. As the author points out at the end of the introduction, the division between the fields of hard sciences and humanities "is not just a cultural problem that we can just wait to be equalized" (p. 8) as it affects the use of technological devices which are increasingly present, even in everyday tasks, such as paying bills or searching addresses, and mediation between devices and humans is precisely the core of HCI.

In Chapter 2, "From Experience to Engagement", the author presents the foundations of the proposed model of engagement, arguing that digital media artefacts are not just products, but have specificities that go beyond their design. Artefacts trigger experiments and experiences are generated by interactions with artefacts. Since artefacts are made by creators, they are the connection between creators and users that generates the experience. At first, the experience was handled through the current studies in HCI, which proved insufficient for the needs of the design, and therefore the research moved from experience to engagement. This chapter explains how the move happened and presents the approach used in the engagement model employed by the book. An important part of this chapter is when the author sets apart the art product from its support through Dewey (1934), for whom "the art product - temple, painting, statue, poem - is not art" but the result of an interaction between the human being and this product, that is, an experience. In order to elucidate us on the question of experience, Zagalo evokes Proust and his madeleines, probably the most famous description of an experience in literature, in his novel, In Search of Lost Time. In this famous narrative, Proust describes in impressive detail an intense nostalgic experience triggered by a single bite on a biscuit with tea. This is the focus of Zagalo's work: the meaning of experience, from which he builds his model in this chapter, and develops it in the next three.

The author has already criticized some models that are still very close to usability studies (Albert, Tullis, \& Albert 2008; Bernhaupt 2009; Hodent 2017; Sauro \& Lewis 2012; Shedroff 2001) that are rooted in metrics and quantifications. He states that "The design goals for creating cultural products are not the same for creating task-oriented products" (p. 17), and in the following chapters $(3,4$ and 5$)$ shows us a description of his model. In Chapter 3, "Engagement Profiles", Zagalo presents us with three types of personalities: abstracters, tinkerers and dramatists, as well as their 
motivations and mental models, in order to delimit the personas for their model. Currently, different systems of psychological profiles, such as the Yee model, the Big Five (Five Factor Model), and the RIASEC (realistic, investigative, artistic, social, enterprising, and conventional), are used to discuss empirical data originated from the motivation of well-known video games, like FarCry, Uncharted, Elder Scrolls, Mass Effect, GTA, Fallout and others. To better develop the proposed profiles, the author relies on six historical figures, two for each mindset, in two different eras: three from the Renaissance, and three from Modernity. The abstracter profile is evidenced by René Descartes (1596-1650) and Stephen Hawking (1942-2018); the tinkerer profile by Leonardo Da Vinci (1452-1519) and Thomas Edison (1847-1931); and the dramatist by William Shakespeare (1564-1616) and Ingmar Bergman (1918-2007). These profiles are then compared with the psychological theories presented and summarized in a large, useful table (p. 52$)$.

In Chapter 4, "Contexts of Engagement", it is time to discuss the environments in which relationships between subjects and artefacts take place in order to define what causes human motivation and curiosity, and how these cognitive processes collaborate to place subjects in the mode of being willing. If the interaction depends on the subjects' will, the main cognitive processes can be curiosity and motivation (Broeck et al. 2010), but the subjects must understand the artefacts. Even if the will is internal to the individual, it is produced through stimuli, through cognitive triggers found in environments around the subjects, which are defined as patterns. In this way, three binaries that encourage human wilfulness are identified (uncertainty/competence; novelty/autonomy; comparison/relatedness) that lead us to three main contexts of action: mastery, creativity and affinity, which can be connected to the 3 profiles defined in Chapter 3: abstracters, tinkerers and dramatists.

Once profiles (Chapter 3) and environments (Chapter 4) have been discussed, the author addresses artefacts and representation. So, Chapter 5, "Artefacts and Representation", starts by working with the concept of representation, based on the phenomenology of C. S. Peirce, the cognitive sciences of J. Bruner, and the art and media creations of Elliot W. Eisner and Edgar Dale. Representation is derived from the representation modes of Bruner and Dale's Cone of Experience: mimetic (iconic), expressive (enactive) and conventional (symbolic), in order to verify their different impacts on interaction. Before closing the chapter, Zagalo presents a discussion on taste and preferences, using Paul Bloom's inverted curve, 
which relates pleasure with familiarity, and the MAYA principle (Most Advanced, Yet Acceptable), thus demonstrating that "the induction of pleasure and the creation of effective experiences are not only built by artefacts, but need to work through the whole communication process people, contexts and artefacts" (p. 108). The analysis of artefacts, therefore, is developed by the analysis of the reasons for pleasure and stimulation and their relationship between the different types of interaction and representation. Some of the games addressed by Zagalo in this chapter are Life is Strange, Spore, Façade, Doom, with everything supported by very illustrative examples, anecdotes, charts and figures.

The time has finally come to see everything working in Chapter 6: "The engagement design model and applied cases". To be honest, here I cheated. By the second chapter I was already anxious to see this model working, so I skipped to this chapter, where we can observe the model in operation through several concrete examples. With my anxiety already appeased, I resumed with Chapters 3, 4, 5, and 6. (If you are anxious like me, I strongly recommend you do this.)

In this chapter, the complete engagement design model is finally presented, showing the entire analysis model developed to work on the research carried out in literature, as well as the empirical data that supports the model. As previously mentioned, the model is based on the relationship of three engagement streams: progression, expression and relation. Each one is presented in contrast to different applications that use its principles, from which several applied cases are analysed in order to understand the model's dimensionality and discrete. Assassin's Creed (2007) is the first case study as an example of multi-engagement, where we see the franchise evolving towards the application of the three streams. Then, the two-by-two flow relationship takes place. For the pair of progression and expression, the games used are SimCity and SimCity 2000; for the pair of relation and expression, The Sims (2004) and sequels; and for the pair of progression and relation, Myst (1993). One of the key elements of the chapter, in addition to seeing the model at work, is how Zagalo gives voice to the players, who are almost always overlooked in game design books and papers. The players' speeches are retrieved from forums and comments and considered for the purposes of data processing and analysis. A problem with the crossing of two streams, relation and progression, is identified, concerning the predisposition in using different mental models in puzzles or narratives, and some clarifications supported by cognitive traits are offered, as follows. Regarding the effort used in interactions, from the difference between 
critical thinking and the problem-solving processes, the author finds that in some games, such as Myst, where these factors run parallel, cognitive overload does not happen due to excessive information, but rather due to the over-processing of the interactor. The industry has treated this problem through regulators of difficulty, easy-medium-difficult, or the new DADs (dynamic difficulty adjustments), and the proposed model also considers this approach. Closing the chapter, the author draws our attention once again to the fact that although the book has mostly presented cases of games, the model applies to other interactive media. The only limits are training games, educational games, serious games and others where the content must direct the form, but it also does not mean that this model cannot be used to increase the interest of the players, or, depending on the case, users.

For anyone interested in game design or interactive media, Zagalo's book is more than important: it is necessary. It is a foray into a major and rarely addressed object: engagement. Far from a walk in the park, this book is as challenging as climbing a mountain, and is worth every minute (or day) invested to reach the top. As usual, some of the theoretical choices or methods can be discussed, but science is built exactly this way: in debate; and a very interdisciplinary approach like that employed by Zagalo will certainly bring about a lot of debate. Zagalo advances on breaking apart the experiences of cultural products from the experiences with task-oriented products, and puts several authors and examples on the table to demonstrate his point of view. The proposed model, of course, seems solid, but it needs more stress testing. I tested the model with two different games (Disco Elysium and Borderlands 3) and the results were very close to those presented in the book. One caveat is an editorial problem in the digitized images of the book, which could have been redesigned or retyped, gaining higher resolution and a more convenient size. As it is an expensive but valuable book, the publishers could have been more careful, even more so in a book that is made to be read several times. As we would say in game design: it is a book of high replay value.

\section{References}

Albert, W., Tullis, T. \& Albert, W. 2008. Measuring the user experience, https://doi.org/10.1016/C2011-0-00016-9 (last access: Dec/02/2020). 
Bernhaupt, R. 2009. Evaluating user experience in games: Concepts and methods. London: Springer-Verlag https://doi.org/10.1007/978-184882-963-3 (last access: Dec/02/2020).

Broeck, A. et al. 2010. "Capturing autonomy, competence, and relatedness at work: Construction and initial validation of the work-related basic need satisfaction scale", Journal of Occupational and Organizational Psychology, 83(4): 981-1002. https://doi.org/10.1348/096317909X481382 (last access: Dec/02/2020).

Dewey, J. 1934. Art as experience. New York: Perigee Books.

Hodent, C. 2017. The Gamer's Brain: How neuroscience and UX can impact video game design.

Boca Raton: CRC Press.

Kelly, K. 2010. What Technology Wants. New York: Viking

Postman, N. 1993. Technopoly: the Surrender of Culture to Technology. New York: Vintage Books/ Kindle Edition.

Sauro, J. \& Lewis, J. R. 2012. Quantifying the user experience: Practical Statistics for User Research. Morgan Kaufmann, https://doi.org/10.1016/C2010-0-65192-3 (last access: Dec/02/2020).

Shedroff, N. 2001. Experience Design. California: Waite Group Press. 